\title{
- Supporting Information - Scalable Synthesis of Janus Particles with High Naturality
}

\author{
Yang Lan, ${ }^{a, b}$ Jingyu Wu, ${ }^{a, c}$ Syung-Hun Han, ${ }^{a, c}$ Sagar Yadavali, ${ }^{a, c}$ \\ David Issadore, ${ }^{c}$ Kathleen J. Stebe, ${ }^{a, *}$ Daeyeon Lee ${ }^{a, *}$ \\ ${ }^{a}$ Department of Chemical and Biomolecular Engineering, University of Pennsylvania, \\ Philadelphia, PA 19104, USA. \\ ${ }^{b}$ Department of Chemical Engineering, University College London, Torrington Place, London \\ WC1E 7JE, United Kingdom. \\ ${ }^{c}$ Department of Bioengineering, University of Pennsylvania, Philadelphia, PA 19104, USA. \\ E-mail:kstebe@seas.upenn.edu; daeyeon@seas.upenn.edu
}

Total number of pages: 7

Total number of figures: 7 


\section{Contents}

S2 Experimental $\quad$ S6

S2.1 Batch-mode synthesis of SBOP/EC Janus particles $\ldots \ldots \ldots \ldots \ldots$. . . . . . . . . .

S2.2 Synthesis of submicrometer-sized SBOP/EC Janus particles $\ldots \ldots \ldots \ldots \ldots$. . . . . . . . 6

S2.3 Fabrication of PDMS microfluidic device . . . . . . . . . . . . . . . . . S6

S2.4 Droplet formation in the PDMS microfluidic device . . . . . . . . . . . . . . S6

S2.5 Fabrication of high-throughput microfluidic device . . . . . . . . . . . . . . S7

S2.6 Droplet formation in the high-throughput microfluidic device . . . . . . . . . . S7

\section{S1 Supplementary data}

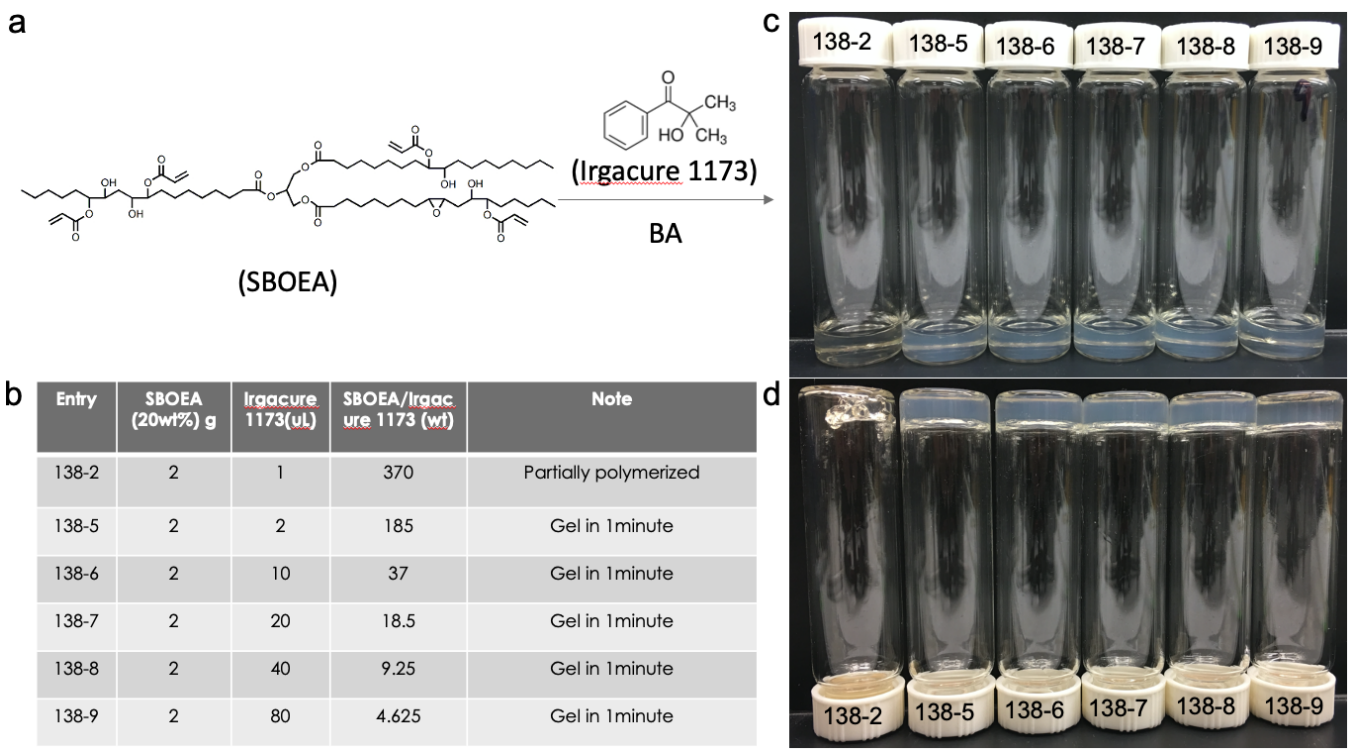

Figure S1: a) Scheme of photo-polymerization of SBOEA in BA. Irgacure 1173 is used as the initiator. b) Summary of the reaction conditions for the photo-polymerization of SBOEA in BA. c and d) Photos of SBOP after the polymerization, showing the gel state of cross-linked SBOP. 


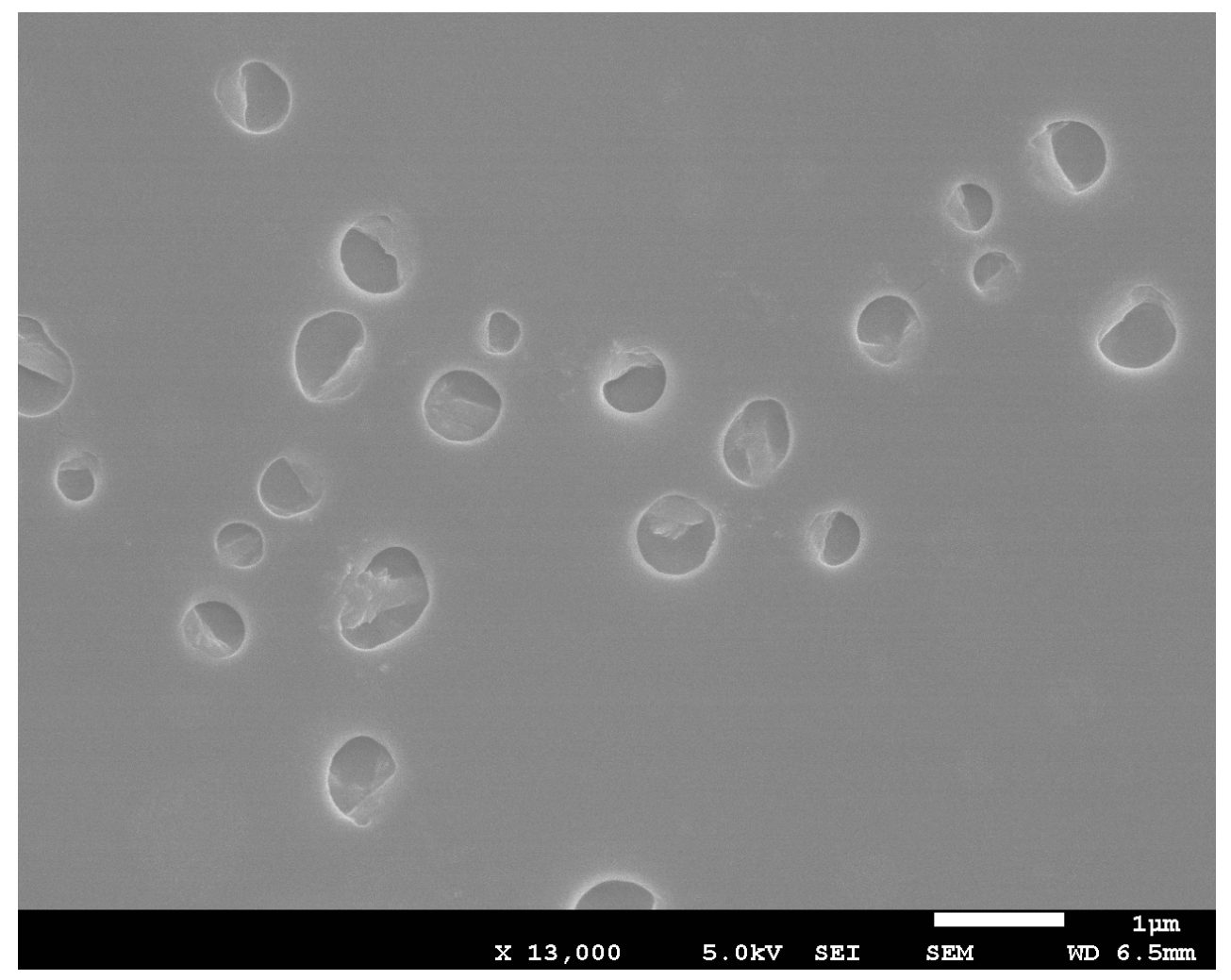

Figure S2: SEM images of submicrometer-sized SBOP/EC Janus particles.

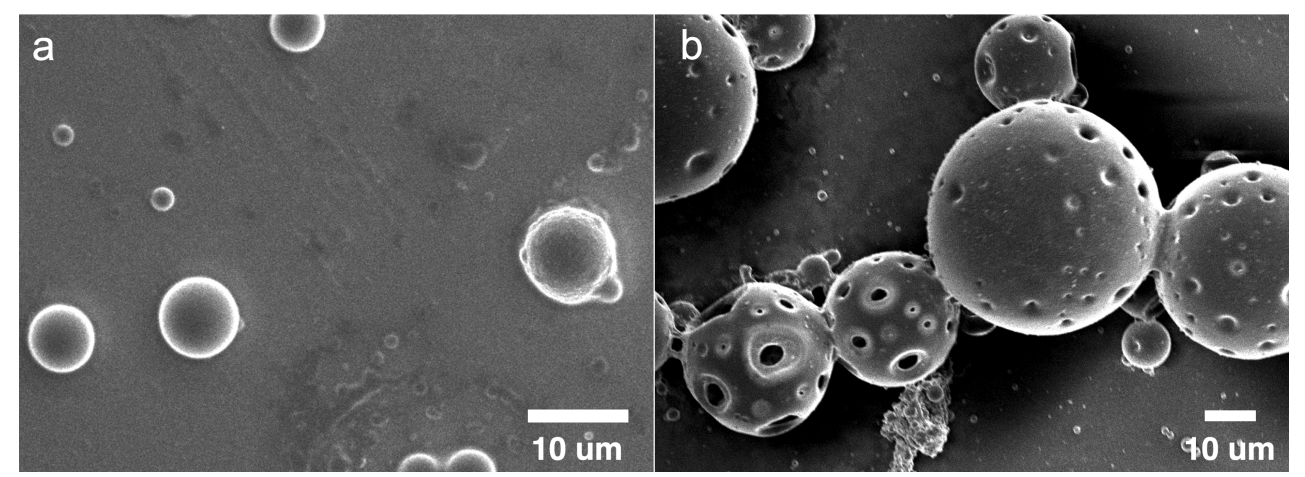

Figure S3: SEM images of particles prepared when SBOEA:EC = 4:1 (a) and SBOEA:EC = 1:4. 


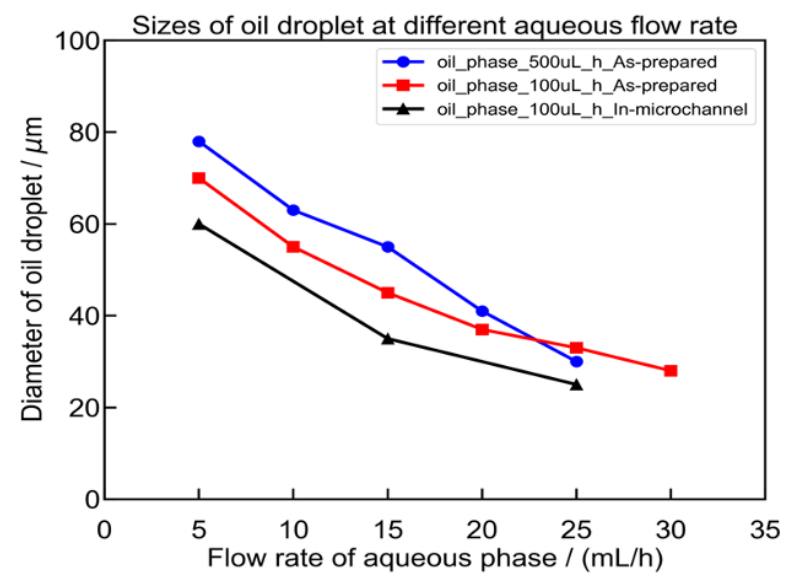

Figure S4: Average diameter of droplets of EC, SBOEA, HMP and BA generated by the flow-focusing microfluidic device at different flow rates. It is worth noting that the emulsion droplets shrink after collecting from the microfluidic device due to mass transfer between the droplets and continuous water phase. Such an exchange likely triggers precipitation of EC in the droplet.
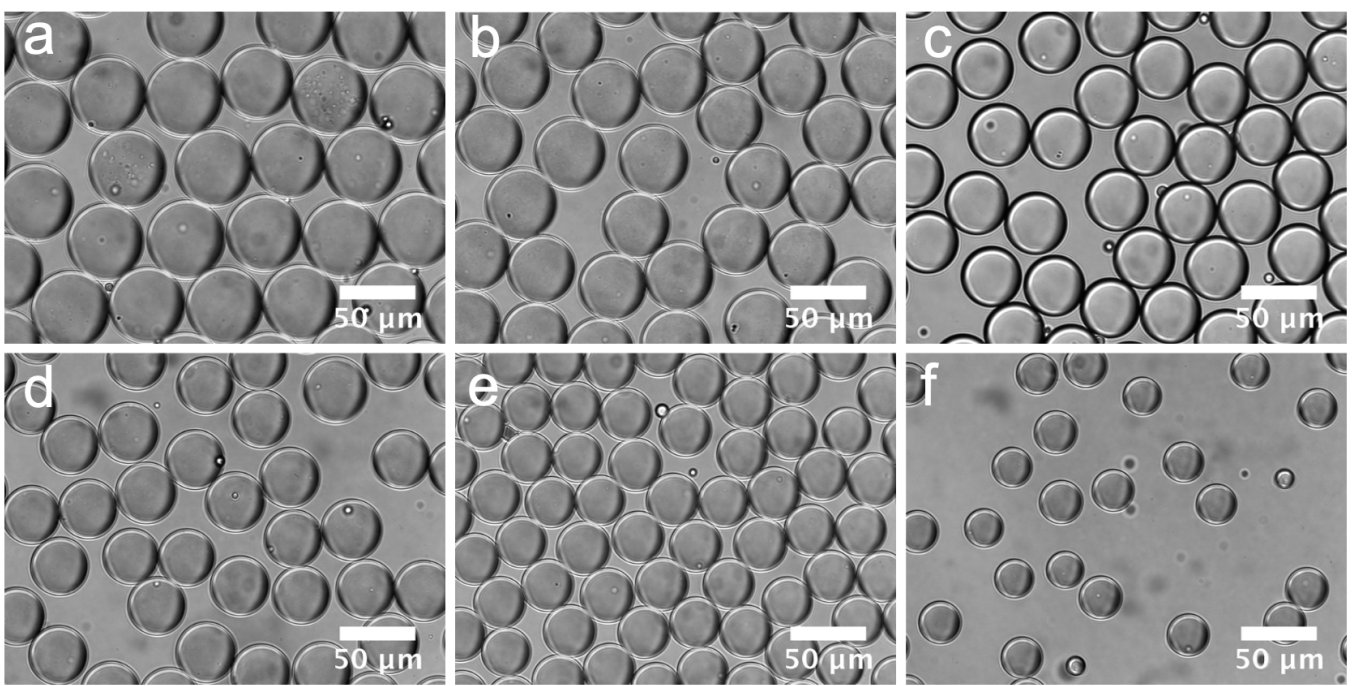

Figure S5: Optical microscopy images of droplet of SBOEA, HMP and BA with an average diameter of a) $50 \mu \mathrm{m}$, b) $45 \mu \mathrm{m}$, c) $40 \mu \mathrm{m}$, d) $35 \mu \mathrm{m}$, e) $30 \mu \mathrm{m}$ and f) $25 \mu \mathrm{m}$. 


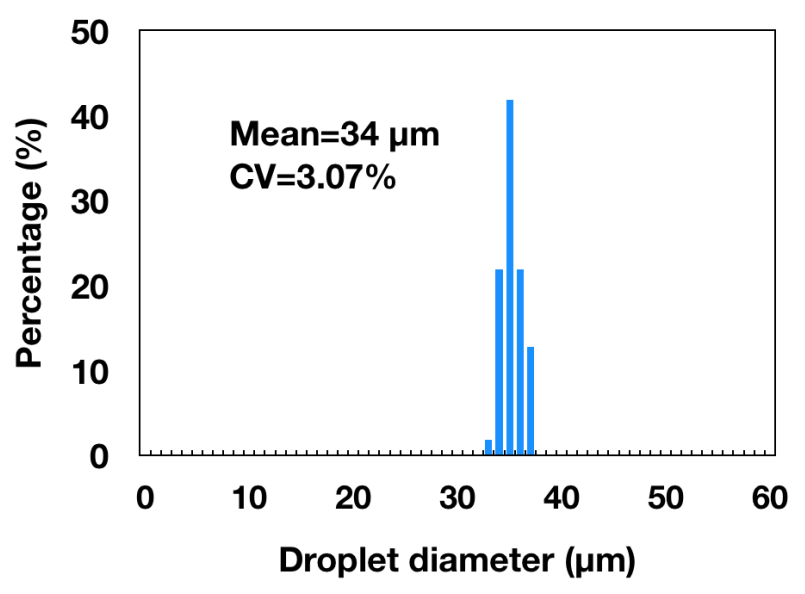

Figure S6: The size distribution of droplets generated by the high-throughput microfluidic device. Conditions: aqueous flow rate is $2.84 \mathrm{~L} / \mathrm{h}$, oil phase flow rate is $1.12 \mathrm{~L} / \mathrm{h}$; aqueous flow pressure is $56 \mathrm{psi}$ and oil phase flow pressure is $24 \mathrm{psi}$. The average diameter of the droplets is $34 \mu \mathrm{m}$ and the coefficient of variation is $3.07 \%$.

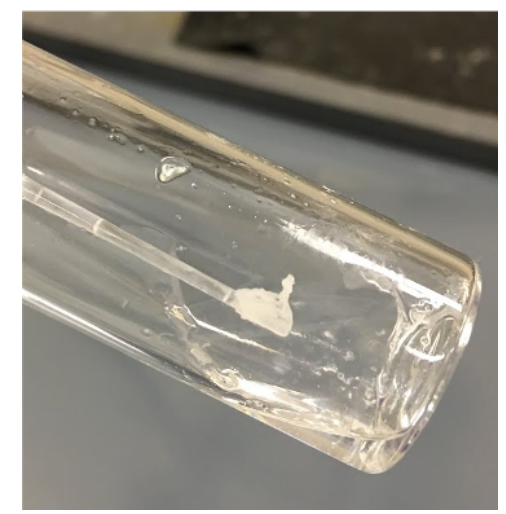

Figure S7: Photo of EC in silicone oil, showing that EC is not soluble in silicone oil. 


\section{S2 Experimental}

All the chemicals are purchased from Sigma Aldrich and used as received unless stated otherwise.

\section{S2.1 Batch-mode synthesis of SBOP/EC Janus particles}

Typically, a mixture of ethyl cellulose (EC, $0.5 \mathrm{~g}$ ), soybean oil epoxidized acrylate (SBOEA, $0.5 \mathrm{~g}$, SBOEA:EC=1:1 wt), butyl acetate (BA, $9.0 \mathrm{~g}$ ) and azobisisobutyronitrile (AIBN, $10.0 \mathrm{mg}$ ) are wellmixed and added dropwise into $90 \mathrm{~mL}$ water (1 wt $\%$ PVA) while stirring vigorously. The mixture is then sealed and stirred at $70{ }^{\circ} \mathrm{C}$ for $24 \mathrm{~h}$. After that, the mixture is further stirred at $70{ }^{\circ} \mathrm{C}$ for $24 \mathrm{~h}$ open to air in a fume hood. The final product is washed with DI water 5 times by centrifugation. When different ratio of SBOEA and EC was used in the synthesis, the overall weight of SBOEA and EC was kept at $1.0 \mathrm{~g}$.

\section{S2.2 Synthesis of submicrometer-sized SBOP/EC Janus particles}

A mixture of ethyl cellulose (EC, $0.5 \mathrm{~g}$ ), soybean oil epoxidized acrylate (SBOEA, $0.5 \mathrm{~g}$ ) and butyl acetate (BA, $9.0 \mathrm{~g}$ ) are added dropwise into $90 \mathrm{~mL}$ water $(1 \mathrm{wt} \%$ PVA and potassium persulfate (KPS, $10.0 \mathrm{mg}$ ) ) while stirring vigorously. The mixture is then sealed and stirred at $70{ }^{\circ} \mathrm{C}$ for $24 \mathrm{~h}$. After that, the mixture is further stirred at $70{ }^{\circ} \mathrm{C}$ for $24 \mathrm{~h}$ open to air in a fume hood. The final product is washed with DI water 5 times by centrifugation.

\section{S2.3 Fabrication of PDMS microfluidic device}

The flow-focusing microfluidic device is prepared via soft lithography by pouring poly(dimethylsiloxane) (Sylgard 184 elastomer kit, Dow Corning, prepolymer : crosslinker $=10: 1)$ onto a silicon wafer patterned with SU-8 photoresist. The PDMS is allowed to solidify at $80^{\circ} \mathrm{C}$ for 4 hours before it is peeled off. The outlets and inlets are generated using a biopsy punch. The enclosed microfluidic channels are formed by attaching the moulded PDMS replica onto microscope slides after exposure to oxygen plasma for 40 $\mathrm{s}$ in a plasma cleaner. To render the device hydrophilic which is suitable for oil-in-water emulsification, $2 \mathrm{wt} \% 3$-(aminopropyl)triethoxysilane aqueous solution was injected into the microchannels and washed away in 20 mins at room temperature. The depth of the microfluidic channel is $50 \mu \mathrm{m}$.

\section{S2.4 Droplet formation in the PDMS microfluidic device}

To generate oil droplets, two different liquids were injected into a microfluidic device, via two syringe pumps (PHD, Harvard Apparatus) with controlled flow rates. In a typical experiment, the oil phase of butyl acetate with $8 \mathrm{wt} \%$ SBOEA, 4 wt $\%$ EC and 0.1 vol\% photo-initiator of 2-hydroxy-2methylpropiophenone (HMP) was used as the dispersed phase. Water with $1 \mathrm{wt} \%$ PVA is used as the continuous phase. The flow rate of the oil phase was kept at $100 \mu \mathrm{L} / \mathrm{h}$ while varying the flow rate of aqueous phase from $500 \mu \mathrm{L} / \mathrm{h}$ to $5000 \mu \mathrm{L} / \mathrm{h}$. 


\section{S2.5 Fabrication of high-throughput microfluidic device}

The highly-parallelized flow-focusing droplet microfluidic device is fabricated similarly to the previous reported method. ${ }^{1,2}$ A double-sided silicon wafer is patterned and etched following a series of lithographic patterning and dry etching to form a three-dimensional microfluidic device. The final microfluidic chip integrated 10,260 droplet generators on a single 4-inch wafer. Briefly, delivery channels are etched for $370 \mu \mathrm{m}$ from one side of the chip using deep reactive ion etching (DRIE). Next, a $6 \mu \mathrm{m}$ silicon dioxide membrane is deposited using plasma-enhanced vapor deposition (PECVD) and patterned as an etch-stop for the latter etching steps on the back side. Through silicon vias (TSVs) are then patterned and etched to connect the fluidic channels on both sides, followed by a final etching of the flow-focusing droplet generators on the back side. The depth of the flow-focusing droplet generators (FFGs) is etched to be 50 $\mu \mathrm{m}$. The three-dimensional etched wafer is sealed by two borosilicate glass wafers using anodic bonding, and eventually bonded to the fluidic connectors with epoxy to form the final device that is ready for use in the experiments.

\section{S2.6 Droplet formation in the high-throughput microfluidic device}

A pressure-driven system is used to operate the high-throughput microfluidic device for droplet generation. Two 1-gallon stainless steel pressure vessels (Alloy products) are pumped by two nitrogen tanks to drive the fluids into the microfluidic device. One vessel is for storing continuous phase, which is water with $1 \mathrm{wt} \%$ PVA, and the other vessel for the dispersed phase, containing butyl acetate with SBOEA, EC and the photo-initiator of 2-hydroxy-2-methylpropiophenone (HMP). The fluidic device is connected to the pressure vessels by PTFE tubings (McMaster Carr 52245K609), and inline filters (McMaster Carr: 9816K72) are used to filter debris in both phases. The flow rates of both phases are controlled by adjusting the pressure valves of the nitrogen tanks and measured by two inline flow meters (McMaster Carr: 5084K23). The flow rate of the dispersed phase was kept at a constant rate of $1.1 \mathrm{~L} / \mathrm{hr}$ while the flow rate of the continuous phase is varied from $1.4 \mathrm{~L} / \mathrm{hr}$ to $3 \mathrm{~L} / \mathrm{hr}$.

\section{References}

[1] H.-H. Jeong, V. R. Yelleswarapu, S. Yadavali, D. Issadore and D. Lee, Lab. Chip, 2015, 15, 43874392.

[2] S. Yadavali, H.-H. Jeong, D. Lee and D. Issadore, Nat. Commun., 2018, 9, 1222-1231. 